\title{
Design and Implementation of Network Resource Management and Configuration System based on Container Cloud Platform
}

\author{
Chen Yuanyuan ${ }^{1, a}$ \\ ${ }^{1}$ Nanchang Institute of Science and Technology, ${ }^{1}$ Nanchang 330108, China \\ achenyuanyuan@126.com
}

Keywords: Cloud platform, Container, Network resource management, Docker, Virtual network

\begin{abstract}
Cloud platform uses virtualization technology to realize management and elastic scaling of the large-scale underlying physical resources. For a long time, the virtual machine has played a role of cloud infrastructure layer backbone, which is used to provide physical resources segregation and control. However, due to additional virtualization, control layer caused additional loss to a cloud platform. Container is similar but a more pinched scale solution, compared with the traditional virtual machine, it uses less resources and less time to start and destroy, to be thought as the better application on a cloud platform release deployment scheme[3]. This is both an opportunity for cloud platform to use the container, but also the challenge of integrated technology. The current container technology is not yet mature in the network, security, storage and other many aspects; there is still a long way to become the construction basis of the cloud platform. This paper analyzes the present network challenges for container cloud platform, and based on the existing research on container network tool, finding that the existing solutions in network isolation, network resource control and elastic extension aspects are very limited far from meeting the needs of cloud platform on network.
\end{abstract}

\section{Introduction}

Cloud computing is a multi-tenant oriented shared resource pool and it can provide computing resources for multiple tenants, and these resources can quickly supply and release according to the requirements of the tenant. These resources include CPU, memory, network, disk, etc., which are the essential condition of the users running on cloud services. Cloud computing means that we need to provide the necessary isolation and resource control for different tenants. Isolation and resource control are two essential requirements to run different tasks on the cloud platform together. Isolation refers to one task cannot affect the operation of other tasks on the same system. In a cloud environment, the isolation is the main performance; in terms of function and safety also have the corresponding isolation requirements. Resource control refers to the constraints of a task to run can use a resource collection. Resources include memory, CPU capacity, disk capacity, etc.as well as the network resources, such as network bandwidth, etc. We control task through using upper limit of memory capacity and time and resource of the CPU etc. For a long time, we complete the cloud platforms opportunely multi-tenant isolation and resource control requirements through the use of the virtual machine. Infrastructure as a Service foot largely became a synonym for the virtual machine. However, the virtual machine needs additional hardware simulation, bringing loss to running performance. There are a lot of studies show the difference of using the virtual machine and bare-metal to performing a task. Isolation and resources based on the technology of container provide a different solution of the virtual machine. Container is not a new technology, Fig. 1 is architecture diagram of Kubernetes of cloud platform. LXC (Linux Container) technology has been existed in the Linux operating system for almost one thousand years, other operating systems also provide similar virtualization technology, such as ADC Workload Partitions, Solaris Container[2]. Although the basic principle to support the container is mature such as the namespace, the Container needs complex configuration with no uniform standard when using, Container technology is not in common use. The development of Docker has brought the Container standard operating environment, like a mirror image of standard specifications and series of Container management tool. 


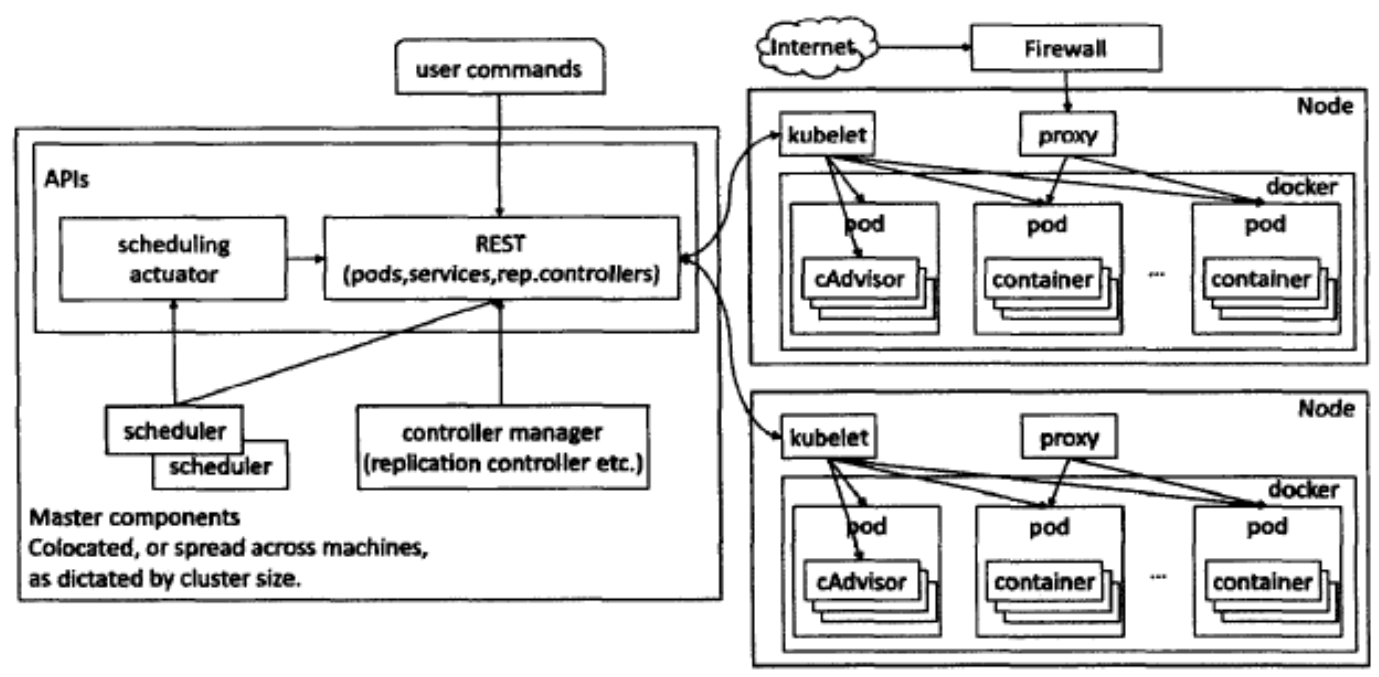

Figure 1: the construction of Kuberntes container cloud platform

\section{Application of configuration system in the network resource management}

As one of the key links of science and technology management system, the needs of configuration management in the daily management work is more and more. Network management and configuration management is one of the core of the management system of science and technology infrastructure, the configuration management is an integral part in a system of perfect network management system. The era of modern information developed, a large number of resources sharing, and requires a more comprehensive, more stable, more advanced network operational system to ensure the normal operation of the various application systems, only by establishing a system of perfect network management system, the comprehensive and centralized management, on the whole network to meet all kinds of business system and application system operational requirements, to provide strong support for all kinds of business smoothly, provides a smooth and efficient operation.

Unified network resource allocation should be brought into the configuration management platform to achieve the centralization, scientific, standardized, and standardization of the network resource allocation management, can effectively help the network maintenance personnel in a timely and accurate understanding of the usage of network resources, assist to implement network operating cost reduction. The realization of the unified network equipment resources configuration repository is the basis of network management system operation, to achieve monitoring view in the network management system, fault management, performance management and change management is of great significance. Unified maintenance of network equipment resources allows the user to definite the content of the network nodes with different changes and input related information, form the maintenance phase of the network change information in detail and stored, to reduce the user changes the maintenance phase of the node definition of error by providing independent maintaining surface. At the same time, the unified network equipment resources library is the main source network reporting system data, setting 24-hour collection each important port and the important node of performance parameters of the data and network fault information and providing different function report to meet the needs of network management to provide a scientific basis can be late for the network construction.

\section{The LXC container virtualization}

Virtualization is the key technology of building a cloud infrastructure indispensable. Cloud computing cloud system, its essence is a large distributed system. Virtualization by in a physical 
platform virtual out more virtual platform, and each of them a virtual platform, can be used as a separate terminal to join the cloud of the distributed system. Compared with the direct use of physical platform, virtualization in the effective use of resources, dynamic allocation has tremendous advantages and high reliability. Platform virtualization is a virtual computer or operating system. The hidden from the user the real computer hardware, show another abstract computing platform [2].Virtual machine to simulate a strong enough hardware to make the client operating system run independently. And the operating system-level virtualization technology based on container key thought lies in the virtual operating system layer according to the requirements of each virtual machine generated a running on physical machines for the copy of the operating system, for each virtual machine to create a good operating system, and implement a virtual machine and its physical machine isolation LXC is short for Linux Containers, is a kind of operating system level virtualization technology based on the container, such as KVM, XEN platform virtualization, LXC uses different method. Platform virtualization is on simulation hardware guide separate virtual system and through the Para virtualization technology mechanism related to reduce overhead. LXC not improves efficiency on the basis of full isolation, but by simple mechanism for isolation, so as to realize the mechanism of system virtualization just like cheroot expansion and transplantation, in the same server at the same time support thousands of the simulation system of [4] as shown in fig. 2, the LXC does not need XEN and KVM virtual machine monitor layer to achieve resource isolation, Shared library on the application of A and B belong to the same container, and C and D belong to another container.

\begin{tabular}{|l|c|c|c|}
\hline App & App & App & App \\
\hline shared library & shared library \\
\hline \multicolumn{3}{|c|}{ host operating system } \\
server \\
\hline \multicolumn{3}{|c|}{}
\end{tabular}

Figure 2: the flow chart

Container will effectively divide the resources managed by a single operating system into isolated group, in order to better balance between the isolated groups have a conflict of resource usage requirements. Compared with other virtualization, Container neither needs instruction level simulation, nor needs to compile instant. Containers can run in local core CPU instructions, and do not need any special explanation mechanism. In addition, it also avoids Para-virtualization and complexity of system calls to replace by providing a way to create and enter the container, the operating system to applications like run on separate machines, but also can share a lot of the underlying resources, just look at the following Fig. 3. 


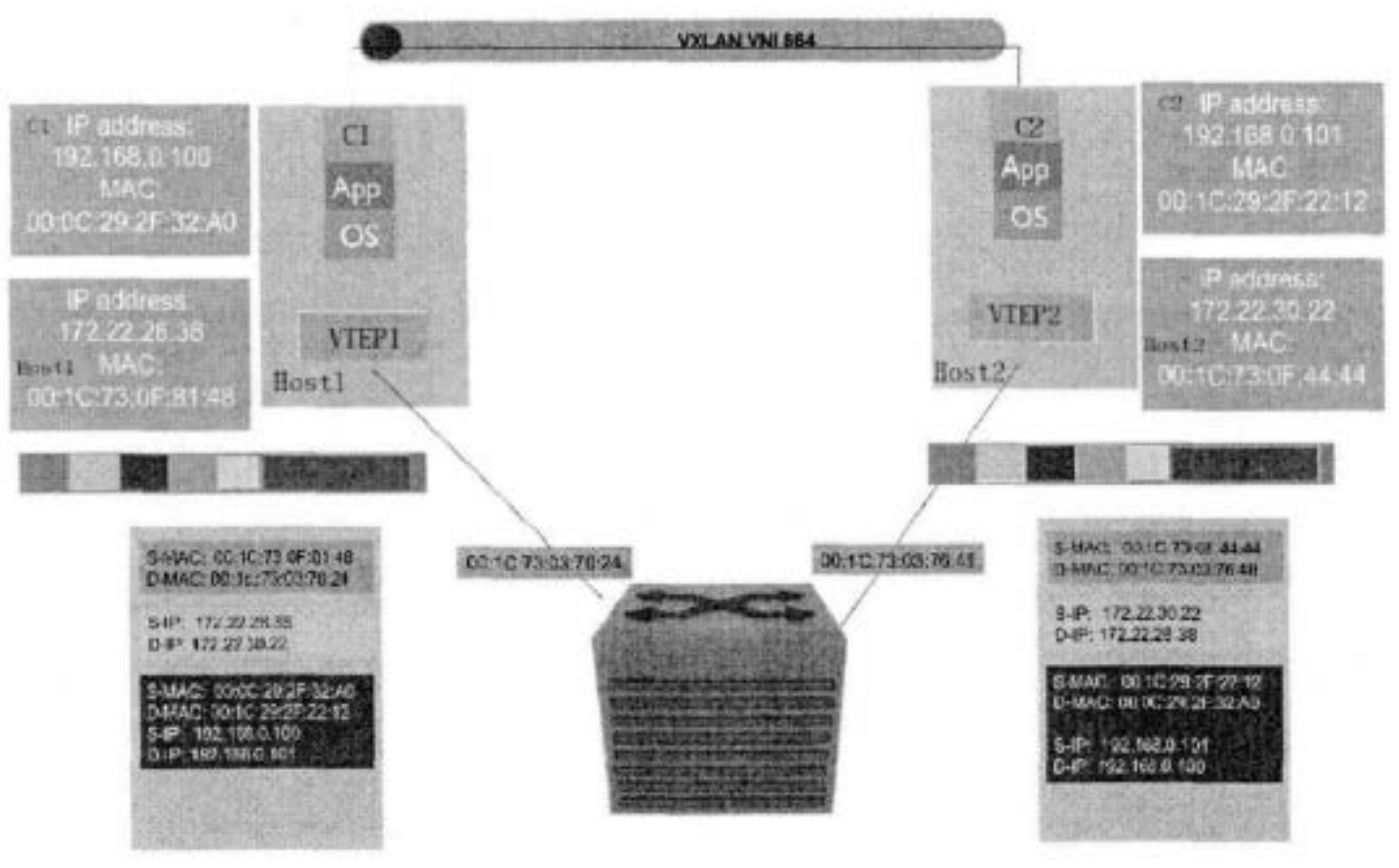

Figure 3: VXLAN database transmit between two different host operating systems

\section{The advantages of Container cloud platform}

The platform abstracts the runtime environment of actual application, eases the burden of the developer deployment operations services, they can focus on the development and application service itself, rather than management network, storage, and plan the specific physical resources etc. Firstly, additional performance loss is small; the cluster resource utilization ascending vessel is the first technical advantages of cloud platform that can increase resource utilization of cloud platform. Low overhead of the container itself makes the single host load too much more computing tasks. The container on the virtual machine can achieve 7 times more than the calculation of the density, it means we can use fewer resources to accomplish the same tasks. Purchasing the server hardware investment is greatly reduced, the system maintenance and software license fee also reduce the potential, the entire cloud platform can greatly improve resource utilization, described for use within the Google cluster management system based on container concludes almost all of the work, such as Search, Google's internal Mail, Map running on the system. Compared with the traditional Iaas based on virtual machine, the system resource allocation granularity is thinner, can decrease additional performance loss, which may save 30\% - 50\% of resources. Secondly, container has smaller image, saves disk space and network transmission time by using different container mirror. Cloud platform can easily support many different programming languages (Java, python, ruby and etc.) and running environment, and these images can be like a base image opportunely, compared with that each virtual machine must have a complete operating system image, so Container would save a lot of disk space. In addition, due to the application service before deployment, need through the network transmission between the host image, smaller mirror means less network transmission time, the speed of deployment of application service also improve greatly. Thirdly, it has faster cluster scale expansion, cloud computing resources saving requirements can be dynamically according to user's requirements and application for and release resources. Destroyed by the container short startup time, so using the container can be faster to meet the needs of the dynamic changes of the application system for resources. For example, when the application system has a lot of requests, it can be started immediately with more containers, responding to the requests a peak level extension; when after the peak, container can also be quickly off idle without saving computational resources, more economical. Finally, the data backup is convenient, easier to migrate a cloud platform using a container storage volume, easily persistent application data and log files. 
Due to one or a few process running inside the container, so compared to the virtual machine, container transfer will be no need to use CRIU tool to save and restore things and easy migration of the vessel.

\section{Summary}

Calculating density is greatly increased after the cloud platform using the container, so as the overall resource utilization. Using containers to build cloud platform is gradually popularized, such as Kubernete and Marathont these open source projects are pioneers. However, the use of containers put forward higher requirements in the aspect of network isolation to the underlying network expansibility and flexibility of cloud platform. This thesis put forward isolation scheme based on VXLAN technology. Compared with the traditional scheme, it supports a number of virtual subnets to meet increasing requirements of cloud platform for virtual subnet. Secondly, we can use Linux Traffic Control module to provide the cloud platforms internet resource control and inspect making up for the industry of the existing network solution without related reading blank skill. For now, the cloud platform based on container and its technology is still in constant development and improvement so that the container community has gained an unprecedented rapid development in the past two years, that more and more developers and companies began to use the container.

Supported by key laboratory of Nanchang city in Jiangxi Province \& characteristic specialty (NGTSZY201001)

\section{References}

[1] H.F. Xue, S.H. Si, H.G.Zhang. The analysis of the virtual machine[J]. Journal of system simulation Journal, 2007, 19 (23) : 5556-5558.

[2] G. Wu. The research of JBS model based on the operating system virtualization technology of containers[J]. Journal of network security technology and application, 2010(4) : 39-41.

[3] S.P. Soltesz. The Container-based on operating system virtualization: a scalable, high performance of the alternative hypervisors [J]. Journal of ACM Operating Systems Review, 2007, 41 (3) : 275-287.

[4] J. Shi. Data mining application under the environment of cloud computing [J]. Micro computer and application, 2015, 34 (5) : 13-15.

[5] J. Wu, Y.J. Zhang. Analysis of dynamic migration optimization of virtual machine[J]. Electronic technology applications, 2015, 41 (11) : 128-131. 